\title{
Spatial variation of the influence of distance from surface water on riparian plant communities in the Okavango Delta, Botswana
}

\author{
Gaolathe Tsheboeng
}

\begin{abstract}
Introduction: Understanding the environmental factors that influence riparian vegetation community composition and distribution is important for understanding wetland ecosystem functioning. This understanding can further serve to inform policy aimed at conserving wetlands such as the Okavango Delta. In the Okavango Delta, information on the influence of distance from surface water on vegetation community composition and structure is limited. The objective of this study was to determine the relationship between distance from surface water and riparian plant communities at different sites in the Okavango Delta.

Methods: Vegetation sampling was conducted in four sites in $20 \mathrm{~m} \times 10 \mathrm{~m}$ belted plots placed perpendicular to the river bank at 0-10 m, 10-20 m, 20-30 m, 30-40 m, and 40-50 m distance classes. At each site, 10-15 plots were sampled at each distance class. Basal area, species richness, cover, and plant diversity were determined for each plot. Indicator species analysis was used to determine the characteristic species at each distance class. One-way analysis of variance and Tukey post hoc analyses were used to compare species diversity, richness, cover, and basal area between distance classes. Generalized linear mixed (GLM) model was used to test for the overall impact of site and distance on each plant parameter.
\end{abstract}

Results: Mean species richness was significantly $(p<0.05)$ higher at 0-10 m than at 20-30 m, 30-40 m, and 40-50 m in Boro. There was no significant difference within other sites. Mean species diversity also varied significantly $(p<0.05)$ in Boro where it was higher at 0-10 m and 10-20 m than at 30-40 m. Mean basal area differed significantly within Jao and Upper Panhandle. In Jao, it was higher $(p<0.05)$ at $0-10 \mathrm{~m}$ than at other distance classes. Mean percentage canopy cover differed significantly within all sites. In addition to this, plant species composition also changed along distance from surface water within each site. From GLM analysis, both distance and site significantly $(p<0.05)$ influenced diversity and basal area. Species richness and cover were significantly $(p<0.05)$ influenced by site only.

Conclusion: Distance from surface water and site are important determinants of riparian plant dynamics in the Okavango Delta.

Keywords: Cover, Okavango Delta, Species richness, Tree and woodland

Correspondence: gtsheboeng@uniswa.sz

Department of Biological Sciences, University of Eswatini, Private Bag 4,

Kwaluseni, Eswatini 


\section{Introduction}

Riparian plant communities, found along river banks, are transition zones between terrestrial and aquatic systems (Naiman and Décamps 1997). In semi-arid environments, riparian plant communities are commonly dynamic showing high habitat heterogeneity and diverse ecological processes and are often rich in plant species diversity (Naiman et al. 2005). The distribution of riparian plant communities is controlled by soil redox conditions, flooding regime, soil chemistry, and light intensity (Everson and Boucher 1998). Surface water availability and fluvial disturbance are also environmental factors that influence plant species composition and distribution along lateral gradients in riparian habitats (Malanson 1993). Flooding disturbance on riparian vegetation decreases with increasing distance from surface water due to an increase of depth to groundwater in the riparian zone (Lite et al. 2005).

Depth to groundwater influences the distribution of plant species, density, and size structure in riparian zones because it determines the availability of a permanent surface water source (Stromberg and Richter 1996). Decline in groundwater may result in a reduction in plant species abundance, cover, productivity, and cause death (Stromberg et al. 2007) while a shallow groundwater table can support high plant species density (Naumburg et al. 2005). Depth to groundwater has been found to increase with increasing distance from surface water in the San Pedro River in the USA (Lite et al. 2005). This has also been found to be the case in the Okavango Delta (McCarthy 2006). Excess water may lead to anoxic conditions in the root zone resulting in stress to tree species due to reduced respiration (Drew 1997). In riparian ecosystems, plant sites close to surface water mostly experience anoxia because of low depth to groundwater resulting in water submerging the roots (Capon 2005). In habitats experiencing high evapotranspiration rates, rainwater may leach salts that accumulate in the soil surface into the groundwater table. If the groundwater table is shallow, the root zone may then be exposed to a saline water layer leading to a reduction in plant growth (Naumburg et al. 2005) due to the harmful effects of salts and anoxic conditions. Conversely, in areas with relatively shallow groundwater (i.e., where plants are using it as a source of water), declining groundwater depth can lead to a reduction in plant species richness and total cover due to a decrease in the availability of a permanent water source to promote primary production (Stromberg and Richter 1996).

Studies in other riparian wetland ecosystems (e.g., Stromberg and Richter 1996; Shafroth et al. 2000; Lite et al. 2005; Stromberg et al. 2007) have shown that distance from surface water through its influence on depth to groundwater is an important environmental variable that influences the distribution and composition of riparian plant communities. In the San Pedro River (USA) riparian plant species tolerant of wet rooting conditions and low depth to groundwater were dominant at sites closer to surface water (ca. $3 \mathrm{~m}$ ) while those that prefer dry conditions and are adapted to greater depth to groundwater were dominant in more elevated sites (ca. 4-8 m) which were found further away from surface water (Stromberg and Richter 1996). An increase in depth to groundwater may lead to a reduction in abundance of riparian woodland species (Shafroth et al. 2000; Baird et al. 2005). However, some riparian plant species have developed morphological adaptations to cope with dry conditions imposed by increased depth to groundwater (Shafroth et al. 2000). These adaptations include tap roots which help them to reach for deep groundwater (Ellery et al. 1993). Due to different tolerance levels, riparian plant species are found in different microsites in the riparian zone. Plant species that are tolerant of shallow depth to groundwater inhabit regularly saturated sites while those that prefer deep depth to groundwater occupy occasionally flooded sites (Damasceno-Junior et al. 2005).

In the Okavango Delta, information on the influence of surface water on riparian plant communities is generally limited. The study by Ellery et al. (1993) found a strong correlation between depth to groundwater and woody plant species distribution in the Okavango Delta, and changes in plant species composition were positively correlated with depth to groundwater and elevation above open water. It was generally found that Diospyros mespiliformis Hochst. EX A.D.C, Garcinia livingstonei T. Anderson, Croton megalobotrys Müll. Arg, and Senegalia nigrescens (Acacia nigrescens Oliv) preferred relatively shallow depth to groundwater while Vachellia erioloba (Acacia erioloba), $V$. tortilis (A. tortilis (Forssk) Hayne), Senegalia mellifera (A. mellifera (Vahl) Benth), and Colophospermum mopane (J. Kirk ex Benth) J. Kirk ex J. Léonard were able to establish and grow where the water depth was greater. However, the study was limited in terms of geographical coverage as it only covered a single island. That study also did not investigate the effect of distance from surface water on riparian plant species composition and distribution.

The recent study by Tsheboeng et al. (2017) investigated the influence of distance from surface water on riparian plant species composition and distribution in the Okavango Delta. However, that study was limited as it did not study response of plant species to distance from surface water in different sites. The aim of the current study was to determine the response of riparian plant communities to distance from surface water in different sites in the Okavango Delta. The Okavango Delta was chosen for this study because it experiences minimum anthropogenic impacts on its water and vegetation resources. As a result, it provides a good case study to investigate the influence of 
distance from surface water on riparian woodland vegetation. It was hypothesized that species richness, diversity, tree height, basal stem area, species richness, and canopy cover will vary with changing distance from surface water in all the sites in the Okavango Delta.

\section{Methods}

\section{Study areas}

The study was conducted in the Okavango Delta. The selected study sites were Boro, Jao, Upper Panhandle (UPH), and Seronga (Fig. 1; Table 1).

The hydrology of the Okavango Delta is characterized by annual flood pulses originating from the Angolan highlands, the peak flows of which generally pass the Mohembo inlet region between March and April, reaching the distal region between May and October (McCarthy 2006). The flood extent in the Delta shows intra- and inter-annual variations (Wolski et al. 2006). The Okavango Delta inflows (flooding) also vary pluri-annually in which there are sequences of years with higher or lower inflows than average (Mazvimavi and Wolski 2006). The Okavango Delta receives mean annual rainfall (between November and February) of approximately $550 \mathrm{~mm}$ (Wilson and Dincer 1976). In the Okavango Delta, groundwater depth varies with distance from surface water such that it is higher and lower in sites closer to and further away from the permanent swamps respectively (McCarthy 2006).

Common woodland species include Vachellia erioloba E. Meyer, Senegalia nigrescens, Croton megalobotrys, Phoenix reclinata Jacq, Diospyros mespiliformis, and Garcinia livingstonei. The soil in the riparian zone is fine sand (Ringrose et al. 2007) resulting in high permeability. The soil salinity in the Okavango Delta is influenced by flooding and transpiration by riparian vegetation (McCarthy and Ellery 1994). During flood peak, the salinity reduces and it increases when water levels are low. This is because the flood waters may dilute the salts (Tsheboeng et al. 2014). Salinity is also high in low flooding frequency floodplains and low in high flooding frequency regions (Bonyongo and Mubyana 2004).

\section{Vegetation sampling}

Four sites were selected for sampling. Riparian woody vegetation was sampled between February 2012 and November 2013. Riparian woody vegetation was sampled in $10 \mathrm{~m} \times 20 \mathrm{~m}$ plots placed perpendicular to the surface water-dry land boundary covering a 50-m distance at $0-10 \mathrm{~m}, 10-20 \mathrm{~m}, 20-30 \mathrm{~m}, 30-40 \mathrm{~m}$, and $40-50 \mathrm{~m}$ intervals. The distance was measured from the current high water line. In each plot, all woody plant species were identified, estimates of canopy cover made following the method by Braun-Blanquet (Ellenberg and Mueller-Dombois 1974), and tree height and stem size measured for each plant species. Stem circumference was measured using a measuring tape at breast height $(1.3 \mathrm{~m})$ while woody plant height was visually estimated. Plant species diversity, richness, basal area, and density were calculated for each distance class. At each site, 75 plots were sampled resulting in a total of 300 plots sampled for the whole study area.

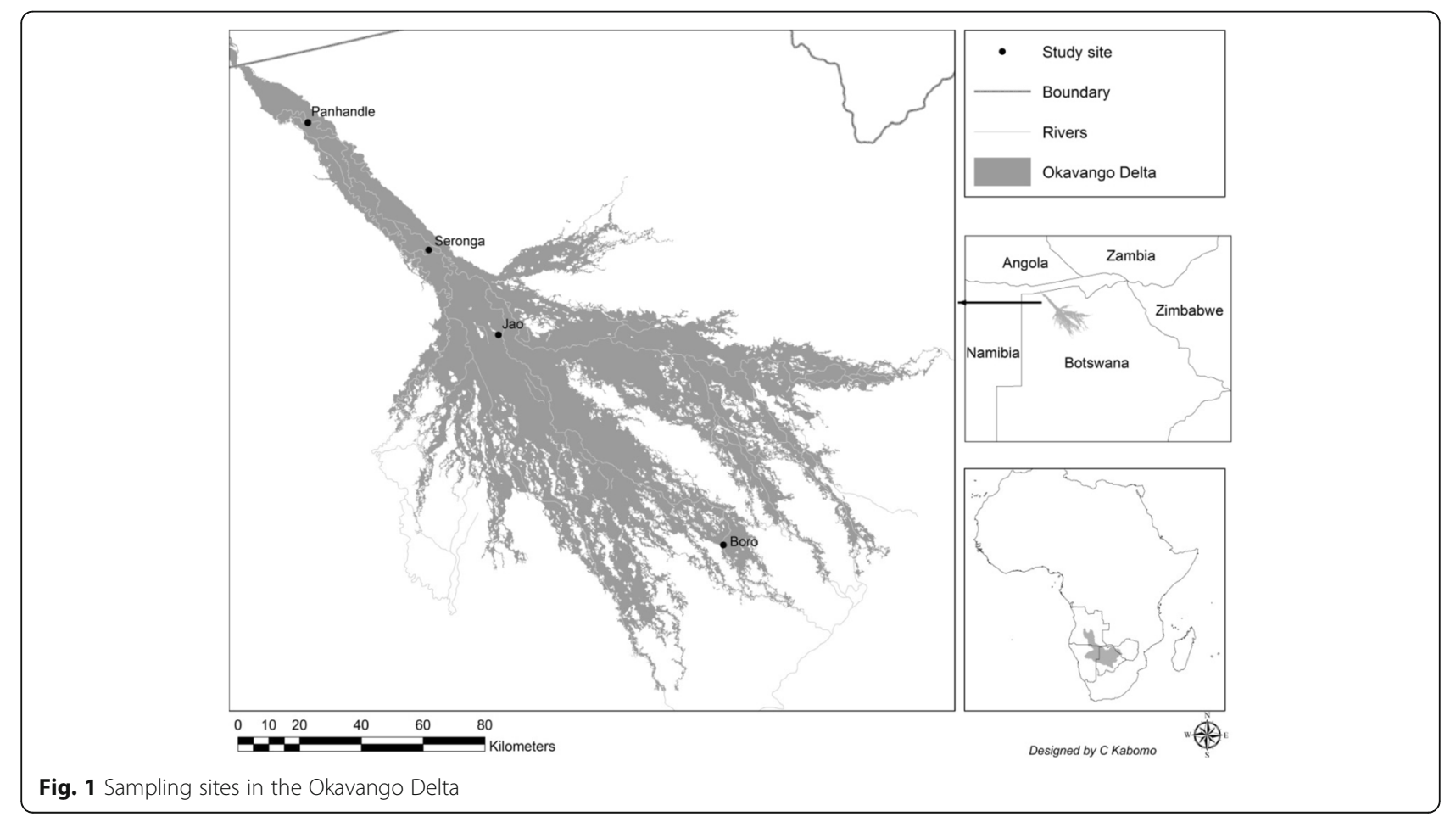


Table 1 Characterization of different sampling sites in the Okavango Delta

\begin{tabular}{llll}
\hline Study area & Coordinates & Location & Common woodland vegetation \\
\hline Boro & $23^{\circ} 9^{\prime} 13^{\prime \prime} \mathrm{E}, 19^{\circ} 32^{\prime} 27^{\prime \prime} \mathrm{S}$ & Lower Delta & Combretum hereroense Schinz, Vachellia erioloba \\
Seronga & $22^{\circ} 17^{\prime} 8^{\prime \prime} \mathrm{E}, 18^{\circ} 48^{\prime} 42^{\prime \prime} \mathrm{S}$ & Upper Delta & $\begin{array}{l}\text { Syzygium cordatum Hochst. ex C Krauss, } \\
\text { Phoenix reclinata Jacq }\end{array}$ \\
Jao & $22^{\circ} 33^{\prime} 40^{\prime \prime} \mathrm{E}$, & S. cordatum, P. reclinata \\
& $-19^{\circ} 1^{\prime} 57^{\prime \prime} \mathrm{S}$ & Upper Delta & \\
Upper Panhandle & $21^{\circ} 52^{\prime} 13^{\prime \prime} \mathrm{E}$, & Upper Delta & Sersia quartiniana A. Rich, Elaeodendron transvaalense \\
& $-18^{\circ} 21^{\prime} 53^{\prime \prime} \mathrm{S}$ & & (Burtt Davy) R.H. Archer \\
\hline
\end{tabular}

\section{Statistical analysis}

Change in woodland species composition in different sites was determined at each distance class based on indicator species analysis (McCune and Grace 2000). Indicator species were determined from mean percentage cover/abundance and relative frequency at each distance class. It was calculated as

Indicator value $=$ total $\%$ species frequency + total $\%$ species abundance

Mean basal area, mean percentage cover, ShannonWeiner diversity index, and mean species richness were calculated for each distance class at each site. Species richness was calculated as the total number of species at each distance class. Diversity was calculated using the Shannon diversity index as:

$$
H^{\prime}=\sum_{i=1}^{S} p i \ln p i
$$

where $\mathrm{H}^{\prime}$ is the Shannon diversity index, $S$ is the total number of species in the community, and $p$ is the proportion of $S$ made up of $i$ th species (Shannon 1948). Basal area $\mathrm{m}^{2} /$ hectare at each distance class was calculated as.

$$
\text { Basal area }=\frac{\text { Sum of the basal area of each tree }}{\text { Area of the sample plot in hectares }}
$$

Mean percentage cover was also determined for five common species at each site. Data were tested for normality using Kolmogorov-Smirnov while homogeneity of variance was tested using the Levene test. After all, the assumptions of normality and homogeneity of variance were met $(p>0.05)$. One-way ANOVA and Tukey post hoc test were used to test for statistically significant differences between these vegetation parameters among distance classes (0-10 m, 10-20 m, 20-30 m, 30-40 m, and 40-50 m).

Generalized linear mixed model analysis was used to test for the overall impact of distance from surface water and site on plant parameters in the Okavango Delta.

\section{Results}

One-way ANOVA showed that there was no significant difference in mean basal area in Boro $(\mathrm{df}=4$, mean square $=0.225, F=1.039, p=0.386)$ and Seronga $(\mathrm{df}=4$, mean square $=0.053, F=0.454, p=0.769$ ). In Jao, Tukey post hoc analysis showed that mean basal area was significantly higher at $0-10 \mathrm{~m}$ than at $10-20 \mathrm{~m}($ mean $=0.28$, $p=0.0001), 20-30 \mathrm{~m}$ (mean $=0.062, p=0.001), 30-40 \mathrm{~m}$ (mean $=0.059, p=0.0001)$, and 40-50 $\mathrm{m} \quad($ mean $=0.074$, $p=0.0001)$. In UPH, it was higher $(\mathrm{df}=4$, mean square $=0.102, F=1.55, p=0.189)$ at $10-20 \mathrm{~m}$ (Fig. 2).

Mean percentage cover differed between the distance classes across all sites. It was significantly higher in $0-10(\mathrm{df}=4$, mean $=9.12, p=0.023)$ and $20-30 \mathrm{~m}(\mathrm{df}=4$, mean $=4.30$ ) in Boro while in Jao, it was marginally significantly higher in $0-10 \mathrm{~m}(\mathrm{df}=4$, mean $=12.50, p=0.055)$ than in 30-40 m ( df =4, mean =6.59) (Fig. 3).

Mean species richness also showed variation between distance classes in different sites. However, that variation was only significant in Boro where it was significantly higher at $0-10 \mathrm{~m}(\mathrm{df}=4$, mean $=7)$ than at $20-30 \mathrm{~m}$ $(\mathrm{df}=4$, mean $=6, p=0.056), 30-40 \mathrm{~m}(\mathrm{df}=4$, mean $=5$, $p=0.0001)$, and $40-50 \mathrm{~m}(\mathrm{df}=4$, mean $=5, p=0.006)$. Mean species richness in Boro was also significantly higher in $10-20 \mathrm{~m}(\mathrm{df}=4$, mean $=6)$ than in $30-40 \mathrm{~m}$ $(\mathrm{df}=4$, mean $=5, p=0.014)$ (Fig. 4).

Mean species diversity was significantly higher at $0-10 \mathrm{~m}$ $(\mathrm{df}=4, F=5.17$, mean $=1.08, p=0.0001)$ and $10-20 \mathrm{~m}$ $(\mathrm{df}=4$, mean $=0.97, p=0.016)$ than at $30-40 \mathrm{~m}(\mathrm{df}=4$, mean $=0.64)$ in Boro $(p<0.05)$. There was no significant difference $(p>0.05)$ in species diversity between distances classes in Seronga $(\mathrm{df}=4$, mean square $=0.105, F=0.300$, $p=0.876)$, Jao $(\mathrm{df}=4$, mean square $=0.119, F=0.664$, $p=0.620)$ and $\mathrm{UPH}(\mathrm{df}=4$, mean square $=0.075, F=0.617$, $p=0.652$ ) (Fig. 5).

Riparian woodland tree species composition and dominant species changed along distance from surface water gradient (Table 2).

In terms of generalized linear mixed model analysis, it was found that diversity and basal area were significantly $(p<0.05)$ influenced by both distance from surface water $(\mathrm{df}=4, p=0.030)$ and site $(\mathrm{df}=3, p=0.030)$. Plant canopy cover $(p=0.054)$ and species richness $(p=0.029)$ were significantly influenced by site only.

\section{Discussion}

There was a variation in species composition along distance from surface water gradient at different sites of Boro, Jao, 

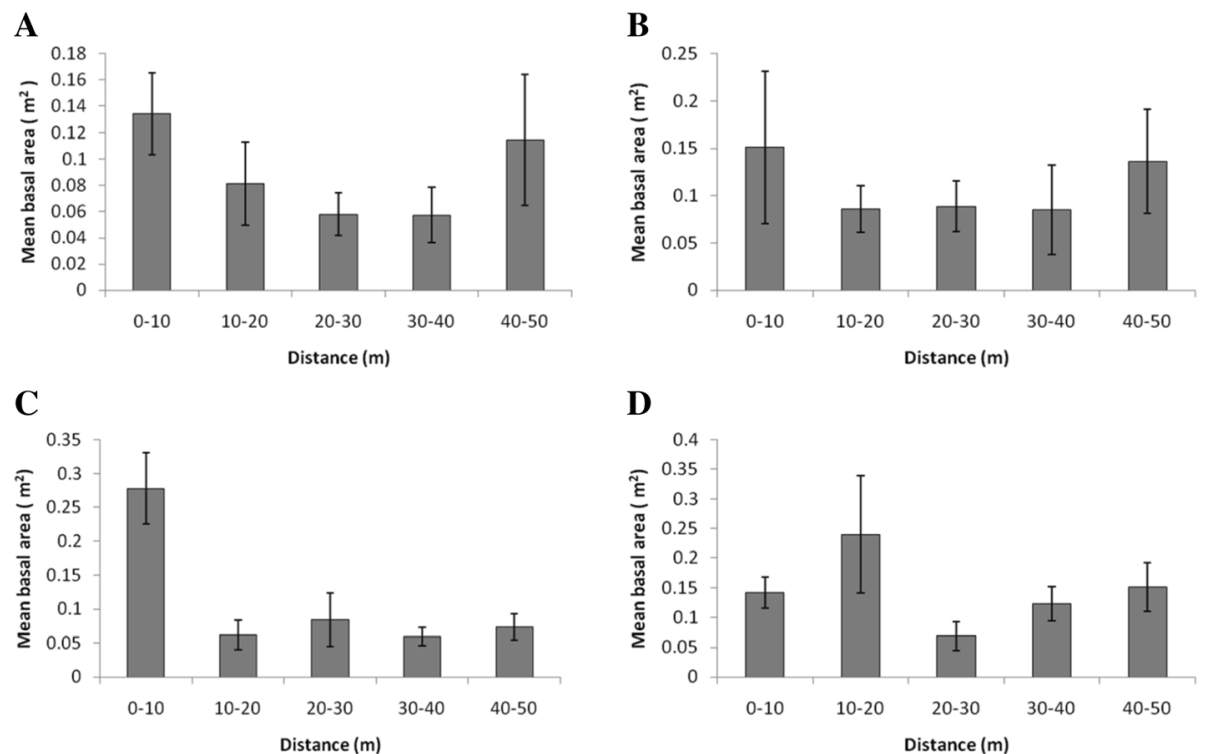

Fig. 2 Mean basal area variation with distance from surface water in Boro (a), Seronga (b), Jao (c), and UPH (d)

Seronga, and Upper Panhandle. Plant species that were found close to surface water at 0-10 m distance class include Croton megalobotrys, Syzygium cordatum, Phoenix reclinata, Searsia tenuinervis, and Garcinia livingstonei. Those that were found far from surface water at 40-50 m distance class include Hyphaene petersiana, Senegalia nigrescens, Combretum imberbe, Vachellia tortilis, and Philenoptera violacea. The distribution of species relative to surface water could be an indication of their tolerance to different moisture regimes. It is expected that sites closer to surface water at 0-10 m will experience more moisture as compared to those that are far from surface water at
40-50 m. This therefore suggests that plant species found close to surface water at $0-10 \mathrm{~m}$ are tolerant of high moisture content as compared to those found at 40-50 m. Furthermore, species close to surface water at 0-10 m are expected to be exposed to shallow groundwater as compared to those far at $40-50 \mathrm{~m}$. This is supported by the findings from Stromberg and Richter (1996), Shafroth et al. (2000), and Baird et al. (2005) who found that woodland plant species tolerant to wet conditions and high groundwater levels are dominant at sites closer to the river bank while those that prefer dry conditions and adapted to low groundwater level dominate sites far from the river

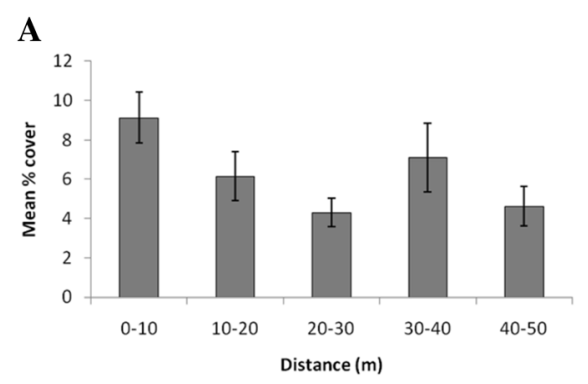

B
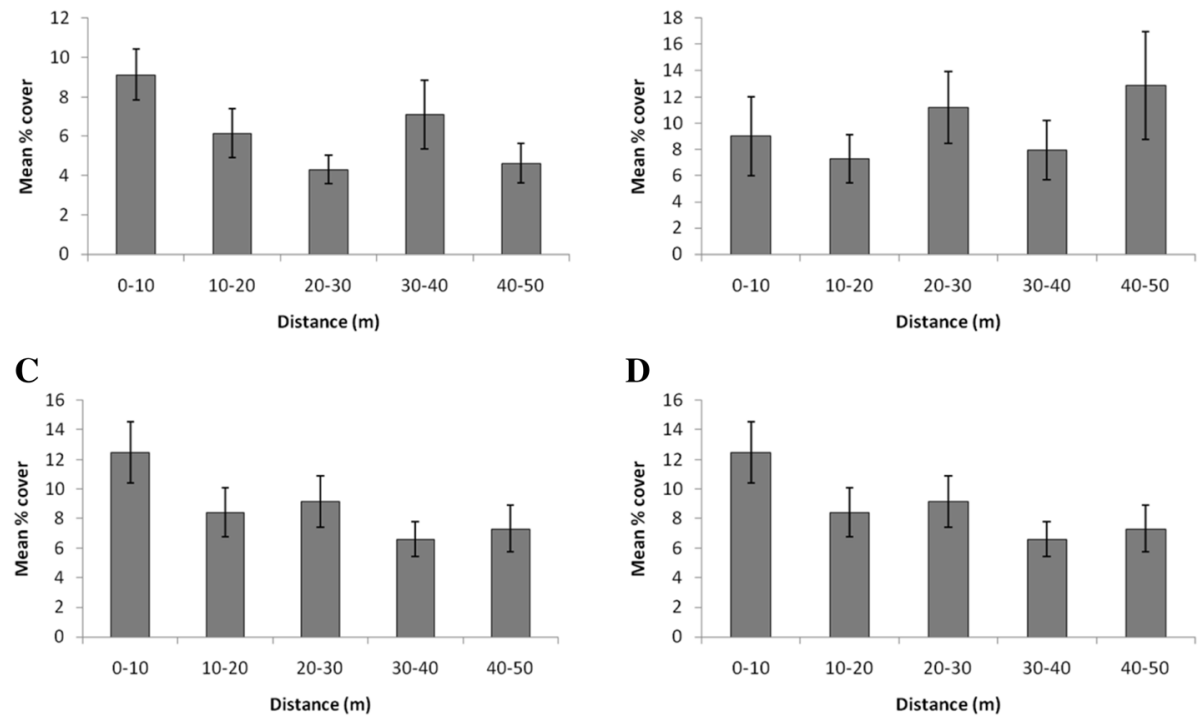

D

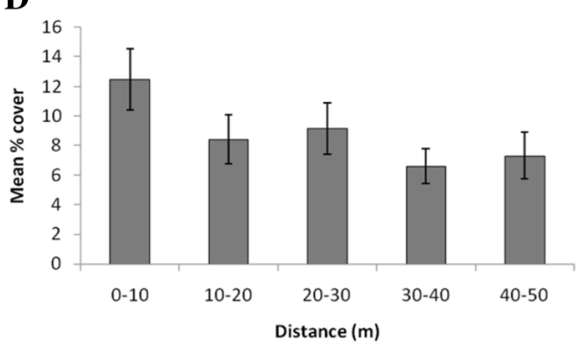

Fig. 3 Mean percentage cover variation with distance from surface water in Boro (a), Seronga (b), Jao (c), and UPH (d) 

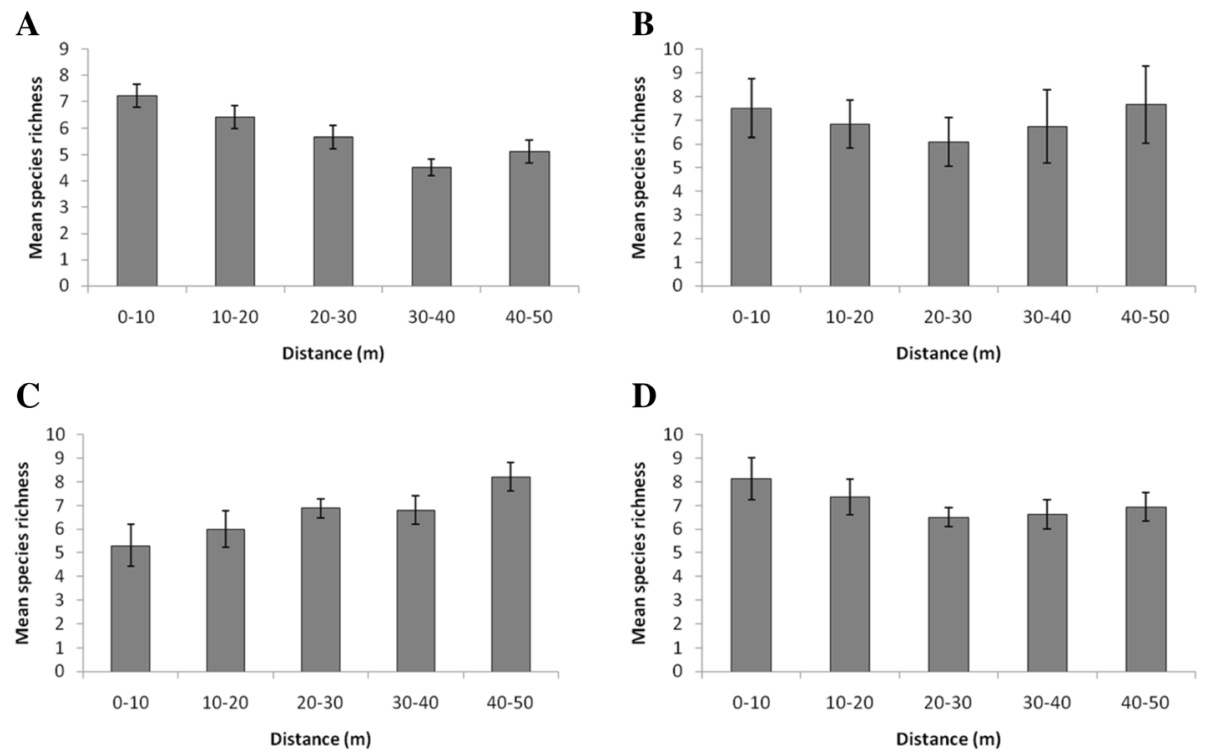

Fig. 4 Mean species richness with distance from surface water in Boro (a), Seronga (b), Jao (c), and UPH (d)

bank. Damasceno-Junior et al. (2005) in the Pantanal, Brazil, also found that flood intolerant species occupied low flooding frequency microsites while flood tolerant species inhabited regularly flooded regions. This was in support of the findings of our study.

In the Okavango Delta, riparian plant species composition and distribution may also be influenced by the intra- and multi-decadal flood variation. The hydrology of the Okavango Delta is characterized by flooding variation which occurs within and between years (Wolski et al. 2006). Due to this variation, some years or series of years receive low floods while others experience high flooding (Wolski et al. 2006). During high and low floods, the groundwater depth becomes shallow and deep respectively (McCarthy 2006). This may have consequences for riparian plant species composition and distribution. The shallow groundwater brings the toxic salts that were initially deposited in the deep layers of the ground within reach of plant roots (McCarthy et al. 1998). Therefore, shallow groundwater may pose a danger to plant survival through salt toxicity and roots submersion which leads to anoxia. This may lead to elimination or exclusion of plants that are not adapted to these new conditions imposed by rising groundwater level. Dead

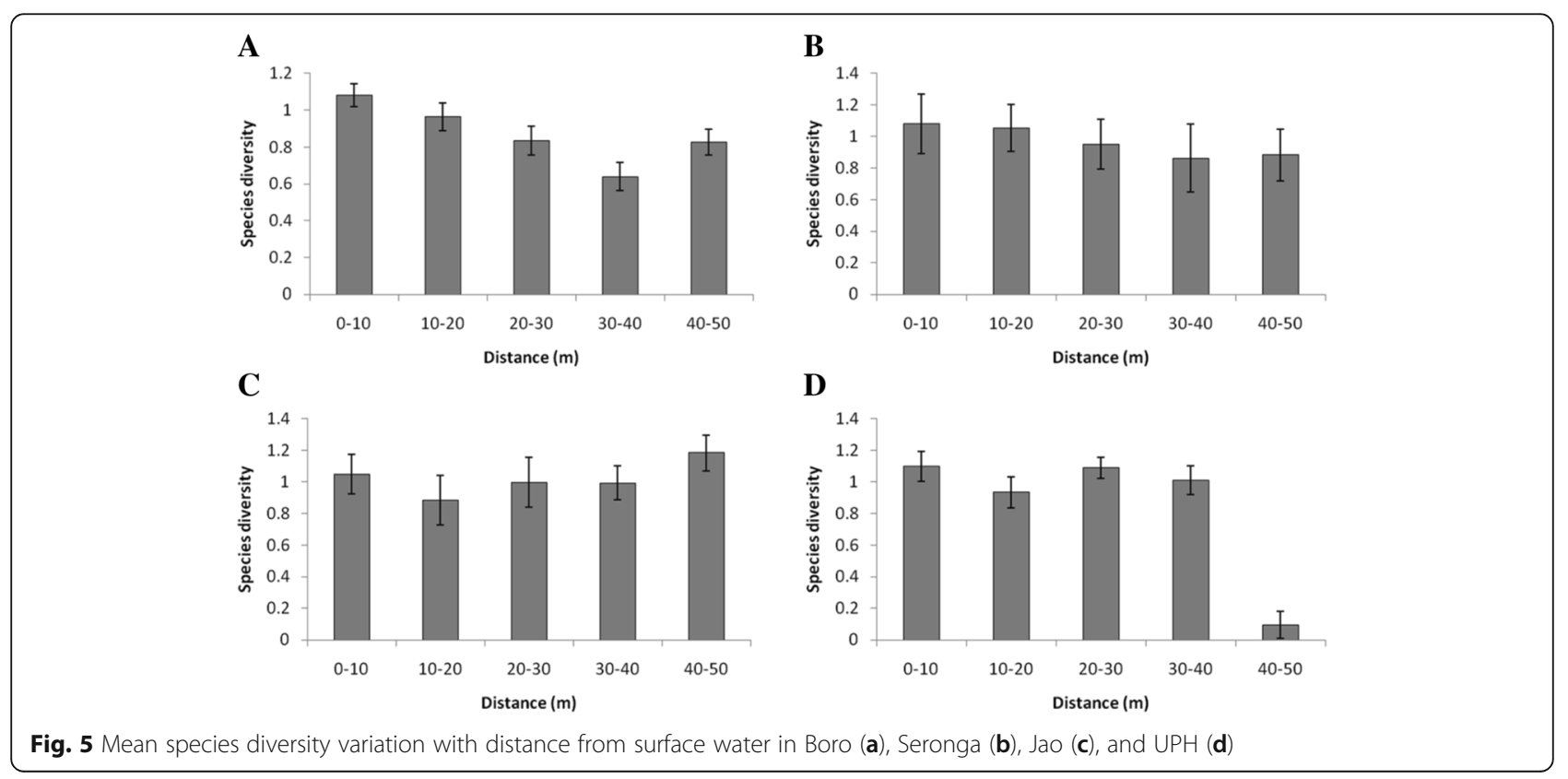


Table 2 Variation in composition of riparian woodland species at each distance class

\begin{tabular}{|c|c|c|c|c|c|}
\hline \multicolumn{6}{|l|}{$\begin{array}{l}\text { Distance } \\
\text { class (m) }\end{array}$} \\
\hline Site & $0-10$ & $10-20$ & $20-30$ & $30-40$ & $40-50$ \\
\hline \multirow[t]{28}{*}{ Boro } & Croton megalobotrys & Croton megalobotrys & Croton megalobotrys & Croton megalobotrys & Croton megalobotrys \\
\hline & Diospyros lycioides & Hyphaene petersiana & Hyphaene petersiana & Hyphaene petersiana & Hyphaene petersiana \\
\hline & Philenoptera violacea & Philenoptera violacea & Philenoptera violacea & Philenoptera violacea & Philenoptera violacea \\
\hline & Hyphaene petersiana & Senegalia nigrescens & Diospyros mespiliformis & Combretum imberbe & Senegalia nigrescens \\
\hline & Kigelia africana & $\begin{array}{l}\text { Combretum } \\
\text { mossambicense }\end{array}$ & Senegalia nigrescens & Diospyros mespiliformis & Combretum imberbe \\
\hline & Diospyros mespiliformis & Gymnosporia senegalensis & $\begin{array}{l}\text { Combretum } \\
\text { mossambicense }\end{array}$ & Kigelia africana & Ficus sycomorus \\
\hline & Gymnosporia senegalensis & Diospyros mespiliformis & Gymnosporia senegalensis & Vachellia hebeclada & $\begin{array}{l}\text { Combretum } \\
\text { mossambicense }\end{array}$ \\
\hline & Combretum imberbe & Kigelia africana & Kigelia africana & $\begin{array}{l}\text { Combretum } \\
\text { mossambicense }\end{array}$ & Vachellia hebeclada \\
\hline & Ficus sycomoros & Euclea divinorum & Diospyros lycioides & Diospyros lycioides & Diospyros lycioides \\
\hline & Senegalia nigrescens & Diospyros lycioides & Ficus sycomorus & Senegalia nigrescens & Euclea divinorum \\
\hline & $\begin{array}{l}\text { Combretum } \\
\text { mossambicense }\end{array}$ & Sclerocarya birrea & Euclea divinorum & Euclea divinorum & Kigelia africana \\
\hline & Euclea divinorum & Combretum imberbe & Combretum hereroense & Garcinia livingstonei & Diospyros mespiliformis \\
\hline & Garcinia livingstonei & Combretum hereroense & Vachellia erioloba & Gymnosporia senegalensis & Gymnosporia senegalensis \\
\hline & Sclerocarya birrea & Garcinia livingstonei & Garcinia livingstonei & Ximenia americana & Albizia harveyi \\
\hline & Ziziphus mucronata & Searsia tenuinervis & Searsia tenuinervis & Vachellia erioloba & Vachellia erioloba \\
\hline & Vachellia hebeclada & Vachellia erioloba & Dichrostachys cinerea & Vachellia tortilis & Searsia tenuinervis \\
\hline & Phyllanthus reticulatus & Phyllanthus reticulatus & Vachellia tortilis & Grewia bicolor & Vachellia tortilis \\
\hline & $\begin{array}{l}\text { Elaeodendron } \\
\text { transvaalense }\end{array}$ & Vachellia tortilis & Albizia harveyi & Dichrostachys cinerea & Dichrostachys cinerea \\
\hline & Vachellia erioloba & Ficus sycomorus & Ximenia americana & Albizia harveyi & Phyllanthus reticulatus \\
\hline & Berchemia discolor & Grewia retinervis & Berchemia discolor & Phyllanthus reticulatus & Combretum hereroense \\
\hline & Combretum hereroense & Albizia harveyi & Grewia bicolor & Berchemia discolor & Garcinia livingstonei \\
\hline & Dichrostachys cinerea & Dichrostachys cinerea & Combretum imberbe & Grewia retinervis & Colophospermum mopane \\
\hline & Grewia flavescens & Ziziphus mucronata & Ziziphus mucronata & Boscia albitrunca & Elaeodendron transvaalense \\
\hline & Vachellia tortilis & Vachellia sieberiana & Vachellia sieberiana & Capparis tomentosa & Grewia flavescens \\
\hline & Albizia harveyi & Berchemia discolor & Grewia flavescens & & Grewia retinervis \\
\hline & Colophospermum mopane & & Vachellia hebeclada & & Boscia albutrinca \\
\hline & & & & & Capparis tomentosa \\
\hline & & & & & Vachellia sieberiana \\
\hline \multirow[t]{8}{*}{ Seronga } & Syzygium cordatum & Phoenix reclinata & Syzygium cordatum & Phoenix reclinata & Syzygium cordatum \\
\hline & Phoenix reclinata & Syzygium cordatum & Phoenix reclinata & Syzygium cordatum & Phoenix reclinata \\
\hline & Diospyros mespiliformis & Senegalia nigrescens & Garcinia livingstonei & Ficus sycomorus & Garcinia livingstonei \\
\hline & Garcinia livingstonei & Diospyros mespiliformis & Senegalia nigrescens & Philenoptera violacea & Diospyros mespiliformis \\
\hline & Vachellia sieberiana & Burkea africana & $\begin{array}{l}\text { Elaeodendron } \\
\text { transvaalense }\end{array}$ & Diospyros mespiliformis & Burkea africana \\
\hline & Senegalia nigrescens & Capparis tomentosa & Ficus thonnigii & Garcinia livingstonei & Albizia harveyi \\
\hline & Searsia quartiniana & Phoenix violacea & Searsia quartiniana & $\begin{array}{l}\text { Elaeodendron } \\
\text { transvaalense }\end{array}$ & Senegalia nigrescens \\
\hline & $\begin{array}{l}\text { Elaeodendron } \\
\text { transvaalense }\end{array}$ & Garcinia livingstonei & Diospyros mespiliformis & Searsia quartiniana & Elaeodendron transvaalense \\
\hline
\end{tabular}


Table 2 Variation in composition of riparian woodland species at each distance class (Continued)

\begin{tabular}{|c|c|c|c|c|c|}
\hline Site & $0-10$ & $10-20$ & $20-30$ & $30-40$ & $40-50$ \\
\hline & Antidesma venosum & Ficus sycomorus & Gymnosporia senegalensis & Senegalia nigrescens & Dovyalis caffra \\
\hline & Ekebergia capensis & Ficus thonnigii & Euclea divinorum & Albizia harveyi & Philenoptera violacea \\
\hline & Dovyalis caffra & $\begin{array}{l}\text { Elaeodendron } \\
\text { transvaalense }\end{array}$ & Capparis tomentosa & Gymnosporia senegalensis & Ficus sycomorus \\
\hline & Kigelia africana & Antidesma venosum & Vachellia sieberiana & Burkea africana & Capparis tomentosa \\
\hline & Euclea divinorum & Euclea divinorum & Philenoptera violacea & Dovyalis caffra & Euclea divinorum \\
\hline & Diospyros lycioides & Ximenia americana & Ficus sycomorus & Antidesma venosum & Searsia quartiniana \\
\hline & Burkea africana & Vachellia sieberiana & Antidesma venosum & Capparis tomentosa & Gymnosporia senegalensis \\
\hline & Gardinia volkensii & Ekebergia capensis & $\begin{array}{l}\text { Combretum } \\
\text { mossambicense }\end{array}$ & $\begin{array}{l}\text { Combretum } \\
\text { mossambicense }\end{array}$ & $\begin{array}{l}\text { Combretum } \\
\text { mossambicense }\end{array}$ \\
\hline & Capparis tomentosa & Combretum imberbe & Dichrostachys cinerea & Euclea divinorum & Antidesma venosum \\
\hline & Ficus sycomorus & Dovyalis caffra & Diospyros lycioides & Searsia tenuinervis & Ximenia americana \\
\hline & Philenoptera violacea & Searsia quartiniana & Ximenia americana & Gardinia volkensii & Searsia tenuinervis \\
\hline & Gymnosporia senegalensis & Albizia harveyi & Phyllanthus reticulatus & Ekebergia capensis & Croton megalobotrys \\
\hline & Croton megalobotrys & Phyllanthus reticulatus & & Croton megalobotrys & Dichrostachys cinerea \\
\hline & Hyphaene petersiana & Croton megalobotrys & & Ximenia americana & Phyllanthus reticulatus \\
\hline & Searsia tenuinervis & Dichrostachys cinerea & & Sclerocarya birrea & \\
\hline & Dichrostachys cinerea & & & Phyllanthus reticulatus & \\
\hline & Phyllanthus reticulatus & & & Diospyros lycioides & \\
\hline & Grewia retinervis & & & & \\
\hline Jao & Syzygium cordatum & Phoenix reclinata & Phoenix reclinata & Garcinia livingstonei & Garcinia livingstonei \\
\hline & Phoenix reclinata & Syzygium cordatum & Garcinia livingstonei & Phoenix reclinata & Phyllanthus reclinata \\
\hline & Garcinia livingstonei & Senegalia nigrescens & Senegalia nigrescens & Senegalia nigrescens & Senegalia nigrescens \\
\hline & Diospyros mespiliformis & Diospyros mespiliformis & Euclea divinorum & Euclea divinorum & Kigelia africana \\
\hline & Ficus sycomorus & Burkea africana & Diospyros mespiliformis & Hyphaene petersiana & Senegalia galpinii \\
\hline & Senegalia nigrescens & Capparis tomentosa & Ficus thonnigii & Capparis tomentosa & Capparis tomentosa \\
\hline & Philenoptera nelsii & Philenoptera violacea & Diospyros lycioides & Ficus thonnigii & Hyphaene petersiana \\
\hline & Diospyros lycioides & Garcinia livingstonei & Berchemia discolor & Diospyros lycioides & Phyllanthus reticulatus \\
\hline & Berchemia discolor & Ficus sycomorus & Ficus sycomorus & Berchemia discolor & Elaeodendron transvaalense \\
\hline & Ficus thonnigii & Ficus thonnigii & Capparis tomentosa & Philenoptera violacea & Ficus sycomorus \\
\hline & Euclea divinorum & Elaeodendron tranvaalense & Gymnosporia senegalensis & Diospyros mespiliformis & Ficus thonnigii \\
\hline & $\begin{array}{l}\text { Elaeodendron } \\
\text { transvaalense }\end{array}$ & Antidesma venosum & Ximenia americana & Gymnosporia senegalensis & Euclea divinorum \\
\hline & Kigelia africana & Euclea divinorum & Hyphaene petersiana & Vachellia karoo & Philenoptera violacea \\
\hline & Capparis tomentosa & Ximenia americana & Philenoptera violacea & Ficus sycomorus & Vachellia karoo \\
\hline & Gymnosporia senegalensis & Vachellia sieberiana & Syzygium cordatum & Croton megalobotrys & Grewia bicolor \\
\hline & Ziziphus mucronata & Ekebergia capensis & Grewia flavescens & Ximenia americana & Grewia flavescens \\
\hline & Searsia tenuinervis & Combretum imberbe & Vachellia karoo & $\begin{array}{l}\text { Elaeodendron } \\
\text { transvaalense }\end{array}$ & $\begin{array}{l}\text { Combretum } \\
\text { mossambicense }\end{array}$ \\
\hline & Phoenix reticulatus & Dovyalis caffra & Searsia tenuinervis & Searsia tenuinervis & Ximenia americana \\
\hline
\end{tabular}


Table 2 Variation in composition of riparian woodland species at each distance class (Continued)

\begin{tabular}{|c|c|c|c|c|c|}
\hline \multicolumn{6}{|c|}{$\begin{array}{l}\text { Distance } \\
\text { class (m) }\end{array}$} \\
\hline Site & $0-10$ & $10-20$ & $20-30$ & $30-40$ & $40-50$ \\
\hline & Hyphaene petersiana & Searsia quartiniana & $\begin{array}{l}\text { Combretum } \\
\text { mossambicense }\end{array}$ & $\begin{array}{l}\text { Combretum } \\
\text { mossambicense }\end{array}$ & Searsia tenuinervis \\
\hline & & Albizia veriscolor & Croton megalobotrys & Phyllanthus reticulatus & Berchemia discolor \\
\hline & & Phyllanthus reticulatus & Phyllanthus reticulatus & Grewia bicolor & Combretum hereroense \\
\hline & & Croton megalobotrys & Kigelia africana & Combretum hereroense & Diospyros mespiliformis \\
\hline & & Dichrostachys cinerea & Sclerocarya birrea & Vachellia sieberiana & Dichrostachys cinerea \\
\hline & & & Eleodendron transvaalense & Dovyalis caffra & Gymnosporia senegalensis \\
\hline & & & & & Croton megalobotrys \\
\hline & & & & & Diospyros lycioides \\
\hline & & & & & Ziziphus mucronata \\
\hline & & & & & Syzygium cordatum \\
\hline & & & & & Phoenix nelsii \\
\hline \multirow[t]{25}{*}{ UPH } & Phoenix reclinata & Phoenix reclinata & Phoenix reclinata & Syzygium cordatum & Phyllanthus reclinata \\
\hline & Syzygium cordatum & Syzygium cordatum & Syzygium cordatum & Phoenix reclinata & Diospyros mespiliformis \\
\hline & Diospyros mespiliformis & Senegalia nigrescens & Garcinia livingstonei & Diospyros mespiliformis & Garcinia livingstonei \\
\hline & Garcinia livingstonei & Garcinia livingstonei & Diospyros mespiliformis & Garcinia livingstonei & Syzygium cordatum \\
\hline & Senegalia nigrescens & Diospyros mespiliformis & Senegalia nigrescens & Senegalia nigrescens & Senegalia nigrescens \\
\hline & Albizia veriscolor & Philenoptera violacea & Philenoptera violacea & Philenoptera violacea & Vachellia tortilis \\
\hline & Phoenix violacea & Combretum imberbe & Croton megalobotrys & Kigelia africana & Philenoptera violacea \\
\hline & Ficus sycomorus & Croton megalobotrys & Berchemia discolor & Croton megalobotrys & Diospyros lycioides \\
\hline & Combretum imberbe & Berchemia discolor & Ficus sycomorus & Vachellia tortilis & Searsia quartiniana \\
\hline & Diospyros lycioides & Ficus sycomorus & Searsia quartiniana & Vachellia erioloba & Gardinia volkensii \\
\hline & Searsia quartiniana & Kigelia africana & Gardinia volkensii & Vachellia sieberiana & Albizia veriscolor \\
\hline & Euclea divinorum & Gymnosporia senegalensis & Diospyros lycioides & Gymnosporia senegalensis & Terminalia sericea \\
\hline & Antidesma venosum & Friesodielsia obovata & Vachellia erioloba & Dichrostachys cinerea & Baphia massaiensis \\
\hline & Friesodielsia obovata & Searsia quartiniana & Capparis tomentosa & Ximenia americana & Dichrostachys cinerea \\
\hline & Croton megalobotrys & Antidesma venosum & Antidesma venosum & Searsia quartiniana & Friesodielsia obovata \\
\hline & Searsia tenuinervis & Euclea divinorum & Gymnosporia senegalensis & Diospyros lycioides & Gymnosporia senegalensis \\
\hline & Gymnosporia senegalensis & Grewia flavescens & Grewia flavescens & Albizia versicolor & Commiphora africana \\
\hline & Gardinia volkensii & Combretum hereroense & Friesodielsia obovata & Friesodielsia obovata & Euclea divinorum \\
\hline & Dichrostachys cinerea & Dovyalis caffra & Dichrostachys cinerea & Antidesma venosum & Croton megalobotrys \\
\hline & Vachellia sieberiana & Gardinia volkensii & Ximenia americana & Capparis tomentosa & Antidesma venosum \\
\hline & Ximenia americana & Diospyros lycioides & Combretum hereroense & Gardinia volkensii & Capparis tomentosa \\
\hline & Berchemia discolor & Ximenia americana & & Grewia flavescens & Elaeodendron transvaalense \\
\hline & $\begin{array}{l}\text { Combretum } \\
\text { mossambicense }\end{array}$ & Phyllanthus reticulatus & & Boscia albutrinca & Grewia flavescens \\
\hline & Capparis tomentosa & $\begin{array}{l}\text { Elaeodendron } \\
\text { transvaalense }\end{array}$ & & Euclea divinorum & Boscia albutrinca \\
\hline & Dovyalis caffra & & & $\begin{array}{l}\text { Combretum } \\
\text { mossambicense }\end{array}$ & Ficus sycomorus \\
\hline
\end{tabular}


Table 2 Variation in composition of riparian woodland species at each distance class (Continued)

\begin{tabular}{|c|c|c|c|c|c|}
\hline \multicolumn{6}{|c|}{$\begin{array}{l}\text { Distance } \\
\text { class (m) }\end{array}$} \\
\hline Site & $0-10$ & $10-20$ & $20-30$ & $30-40$ & $40-50$ \\
\hline & \multirow[t]{9}{*}{ Ekebergia capensis } & & & $\begin{array}{l}\text { Elaeodendron } \\
\text { transvaalense }\end{array}$ & Searsia tenuinervis \\
\hline & & & & Searsia tenuinervis & Ximenia americana \\
\hline & & & & Hyphaene petersiana & Vachellia seiberiana \\
\hline & & & & Ficus sycomorus & Dovyalis caffra \\
\hline & & & & Phyllanthus reticulatus & Hyphaene petersiana \\
\hline & & & & Combretum hereroense & Vachellia erioloba \\
\hline & & & & Dovyalis caffra & Berchemia discolor \\
\hline & & & & Terminalia sericea & $\begin{array}{l}\text { Combretum } \\
\text { mossambiecense }\end{array}$ \\
\hline & & & & & Combretum hereroense \\
\hline
\end{tabular}

individuals of Croton megalobotrys, Senegalia nigrescens, Diospyros mespiliformis, and Philenoptera violacea were observed in this study, and their death could be an indication of their inability to withstand rising groundwater depth and salinity. Conversely, during low flood, the groundwater may be too deep to be reached by plant roots resulting in water stress. Only plants that are tolerant of dry conditions through development of tap roots to reach for the deep groundwater layers and shedding of their leaves to minimize water loss may survive while those that are intolerant of dry conditions may be eliminated. This may explain the variation in riparian species composition along distance from surface water found in the Okavango Delta (Table 2).

Species richness varied significantly with distance from surface water in all the sites. This is in agreement with Lite et al. (2005) who also observed variation in species richness in the San Pedro River with increasing distance from surface water. It implies that different species establish along the groundwater depth gradients to which they are adapted along distance from surface water gradient in both the Okavango Delta. Salinity tolerance may also determine species richness along the distance from surface water gradient. McCarthy (2006) found that as the distance from surface water increases, salinity levels also increase in the Okavango Delta. This leads to woodland species tolerant of salinity and deep groundwater table colonizing areas further away from the surface water. In this study, distribution of species could be influenced by salinity gradients along the distance from surface water continuum. For instance, in Boro, species that are tolerant to saline conditions such as Hyphaene petersiana and Combretum imberbe (Ringrose et al. 2007) were found in the 40-50 m distance class which was associated with high salt levels. This is consistent with Ellery et al. (1993) who observed plant zonation along a salinity gradient in the Okavango Delta where the fringes of the islands with low salinity levels were colonized by riparian woodland species of Syzygium cordatum, Ficus sycomorus, Phoenix reclinata, Garcinia livingstonei, and Diospyros mespiliformis. The island interiors with relatively higher salinity levels than the fringes were colonized by Croton megalobotrys, Senegalia nigrescens, and Hyphaene petersiana with the center of the islands with overall high salt levels dominated by non-woody species of Sporobolus spicatus (Vahl) Kunth. This zonation suggests that each distance class from surface water, different woody species have different levels of salinity which they can tolerate and beyond which they may be completely excluded.

The current study has also indicated that mean basal area significantly varied with distance from surface water across different sites. It was high at $0-10 \mathrm{~m}$ and $40-50 \mathrm{~m}$ in Boro and Seronga and significantly higher at $0-10$ and $10-20 \mathrm{~m}$ in Jao and UPH respectively. High mean basal area in Boro at sites closer to the permanent swamp and those that were at $40-50 \mathrm{~m}$ could be an indication that depth to groundwater at this distance from surface water in these sites was suitable for the establishment of large woody species. Examples of these species found at 0-10 $\mathrm{m}$ in the Boro system include Diospyros mespiliformis, Ficus sycomorus, P. violacea, and Garcinia livingstonei which have contributed to large basal area. In Seronga, species which contributed to the larger basal area at $0-10 \mathrm{~m}$ were Syzygium cordatum, D. mespiliformis, and G. livingsto$n e i$. These species are tolerant of shallow groundwater conditions (Ellery et al. 1993) which may explain their establishment at $0-10 \mathrm{~m}$ as this distance class is likely to be characterized by shallow groundwater depth since it is close to surface water.

Mean plant species diversity was significantly higher at $0-10 \mathrm{~m}$ and $10-20 \mathrm{~m}$ than at $30-40 \mathrm{~m}$ in Boro while there was no significant variation within other sites. High 
species diversity at these distance classes may imply that there was co-existence of species tolerant of high and low depth to groundwater (Huston 1979) as a result of changing flooding magnitude during both short and long periods. During high floods, species that do not prefer permanent flooding and those that are tolerant of it may co-exist. The co-existence of these two sets of species may lead to an increase in species diversity as predicted by the intermediate disturbance hypothesis. The hypothesis predicts that at intermediate disturbance levels, species diversity increases due to the co-existence of both competitive and opportunistic species (Huston 1979). However, diversity may eventually decrease if flooding in the riparian zone occurs for a duration long enough to kill the species that are intolerant of flooding. Conversely, if there is long-term drying and high depth to groundwater in the riparian zone, species that prefer wet conditions will be excluded resulting in a decrease in species diversity.

In the Boro and Seronga systems, the $0-10 \mathrm{~m}$ distance class was colonized by species such as $P$. violacea, $D$. mespiliformis, G. livingstonei, and F. sycomorus which prefer shallow groundwater; Ziziphus mucronata, Vachellia hebeclada, Senegalia nigrescens, and Dichrostachys cinerea which are tolerant of deep depth to groundwater; and Combretum imberbe and Hyphaene petersiana adapted to saline groundwater conditions (Ringrose et al. 2007). Therefore, the co-existence of these species with different preferences of groundwater chemistry and depth may have led to an increase in diversity at $0-10 \mathrm{~m}$ in Boro and Seronga. Jao had significantly high species diversity at 40-50 m while in UPH, diversity was significantly lower at this distance class. This was because of species at 40-50 m which tolerated dry conditions and resulted in high species diversity. Some of these species were Senegalia galpinii, S. nigrescens, Grewia bicolor, D. cinerea, and $P$. nelsii which were excluded from other distance classes probably due to unfavorable flooding and groundwater conditions. Conversely, in UPH, species diversity was significantly lower at $40-50 \mathrm{~m}$ distance class. This is because it was dominated by species that are mostly tolerant of deep groundwater table while those that prefer shallow groundwater were excluded. Conversely, other distance classes in UPH had species that were tolerant of both high and low groundwater tables co-existing which resulted in high diversity suggesting that they may have experienced intermediate disturbance levels from flooding. This is in agreement with Pollock et al. (1998) who also found that there was an increase in species diversity at sites that experienced intermediate levels of flooding disturbance. Generally, the consistency of these study results with those of other wetlands elsewhere suggests that functionally these ecosystems are similar in terms of the influence of hydrology on vegetation communities.

\section{Conclusions}

This study showed that riparian woodland characteristics differed significantly with distance from surface water within and between in all the sites. As the distance from surface water increases, it is expected that depth to groundwater also increases. As a result, different species establish in microsites to which they are adapted along the gradient of distance from surface water. These results suggest that plant species composition, basal area, and species richness may be used as indicators of the hydrologic status of a given site in the Okavango Delta. This will be useful in areas where the information on that is not available.

\section{Abbreviations \\ GLMM: Generalized linear mixed model; UPH: Upper Panhandle}

\section{Acknowledgments}

This work was carried out under Research Permit Number EWT: 8/36/4 XXVIII (10) issued by the Ministry of Environment, Wildlife and Tourism, Botswana. I would like to thank the German Federal Ministry for Education and Research (BMBF) who sponsored this study through The Future Okavango (TFO) project. The author would also like to thank Mr. M. Mmusi and Mr. W. Khaneguba who assisted in data collection and the staff at the Peter Smith Herbarium for helping with plant identification.

\section{Funding}

This study which is part of GT's PhD work was sponsored by the German Federal Ministry for Education and Research (BMBF). The funder did not participate in either the design of the study or data collection and write up.

\section{Authors' contributions}

GT designed the study. GT collected the data analyzed it and compiled the manuscript. The author read and approved the final manuscript.

\section{Author's information}

GT is a Lecturer of Ecology at the University of Eswatini. His research interests are in wetland plant ecology, impact of land use on vegetation, and bush encroachment in savanna ecosystems.

Ethics approval and consent to participate

Not applicable

Consent for publication

Not applicable

\section{Competing interests}

The author declares that there are no competing interests.

\section{Publisher's Note}

Springer Nature remains neutral with regard to jurisdictional claims in published maps and institutional affiliations.

Received: 22 February 2018 Accepted: 13 August 2018

Published online: 29 August 2018

\section{References}

Baird KJ, Stromberg JC, Maddock T (2005) Linking riparian dynamics and groundwater: an eco-hydrologic approach to modelling ground water and riparian vegetation. Environ Manag 36:551-564

Bonyongo MC, Mubyana T (2004) Soil nutrient status in vegetation communities of the Okavango Delta floodplains. S Afr J Sci 100:337-340

Capon SJ (2005) Flood variability and spatial variation in plant community composition and structure on a large arid floodplain. J Arid Environ 60: 283-302.

Damasceno-Junior GA, Semir J, Dos Santos FAM, Leitão-Filho HF (2005) Structure, distribution of species and inundation in a riparian forest of Rio Paraguai, Pantanal, Brazil. Flora 200:199-135 
Drew MC (1997) Oxygen deficiency and root metabolism: injury and acclimation under hypoxia and anoxia. Annu Rev Plant Biol 48:223-250

Ellenberg H, Mueller-Dombois D (1974) Aims and methods of vegetation ecology. Wiley, New York

Ellery WN, Ellery K, McCarthy TS (1993) Plant distribution in islands of the Okavango Delta, Botswana: determinants and feedback interactions. Afr J Ecol 31:118-134

Everson DA, Boucher DH (1998) Tree species-richness and topographic complexity along the riparian edge of the Potomac River. Forest Ecol Manag 109:305-314

Huston M (1979) A general hypothesis of species diversity. American Nat 113:81-101

Lite SJ, Bagstad KJ, Stromberg JC (2005) Riparian plant species richness along lateral and longitudinal gradients of water stress and flood disturbance, San Pedro River, Arizona, USA. J Arid Environ 6:785-813

Malanson GP (1993) Riparian landscapes. Cambridge University Press, New York Mazvimavi D, Wolski P (2006) Long-term variations of annual flows of the Okavango and Zambezi Rivers. Phys Chem Earth 31:944-951

McCarthy T, Ellery WN (1994) The effect of vegetation on soil and ground water chemistry and hydrology of islands in the seasonal swamps of the Okavango fan, Botswana. J Hydrol 154:169-193

McCarthy TS (2006) Groundwater in the wetlands of the Okavango Delta, Botswana and its contribution to the structure and function of the ecosystem. J Hydrol 320:264-282

McCarthy TS, Bloem A, Larkin PA (1998) Observation on the hydrology and geohydrology of the Okavango Delta. S Afr J Geol 101:101-107

McCune B, Grace JB (2000) Analysis of ecological communities. MjM Software, Gleden Beach

Naiman RJ, Bechtold JS, Drake DC, Latterell JJ, O'Keefe TC, Balian EV (2005) Origins, patterns and importance of heterogeneity in riparian systems. In: Lovett MG, Turner MG, Jones CG, Weathers KC (eds) Ecosystem function in heterogenous landscapes. Springer, New York, pp 279-309

Naiman RJ, Décamps H (1997) The ecology of interfaces: riparian zones. Annu Rev Ecol Syst 28:621-658

Naumburg E, Mata-Gonzalez R, Hunter RG, Mclendon T, Martin DW (2005) Phreatophytic vegetation and groundwater fluctuations: a review of current research and application of ecosystem response modelling with an emphasis on Great Basin vegetation. Environ Manag 35:726-740

Pollock MM, Naiman RJ, Hanley TA (1998) Plant species richness in forested and emergent wetlands-a test of biodiversity theory. Ecol 79:94-105

Ringrose S, Vanderpost C, Matheson W. Huntsman-Mapila P, Murray-Hudson M, Jellema A (2007) Indicators of desiccation driven change in the distal Okavango Delta, Botswana. J Arid Environ 68:88-112

Shafroth PB, Stromberg JC, Patten DT (2000) Woody riparian vegetation response to different alluvial water table regimes. West N Am Nat 60:66-76

Shannon CE (1948) A mathematical theory of communication. Bell Syst Tech J 27: $379-423$

Stromberg JC, Beauchamp VB, Dixon MD, Lite SJ, Paradzick C (2007) Importance of low-flow and high-flow characteristics to restoration of riparian vegetation along rivers in arid south-western United States. Fresh water Biol 52:651-679

Stromberg JC, Richter B (1996) Effects of ground water decline on riparian vegetation of semi-arid regions: the San Pedro, Arizona. Ecol Appl 6:113-131

Tsheboeng G, Bonyongo M, Murray-Hudson M (2014) Flood variation and soil nutrient content in floodplain vegetation communities in the Okavango Delta. S Afr J Sci 110:1-5

Tsheboeng G, Murray-Hudson M, Kashe K (2017) Response of riparian plant communities to distance from surface water in the Okavango Delta, Botswana. Afr J Ecol 55:402-410

Wilson BH, Dincer T (1976) An introduction to the hydrology and hydrography of the Okavango Delta. Proceedings of the symposium on the Okavango Delta and its future utilization held in Gaborone, Botswana

Wolski P, Savenije HHG, Murray-Hudson M, Gumbricht M (2006) Modelling of the flooding in the Okavango Delta, Botswana, using a GIS model. J Hydrol 331:58-72

\section{Submit your manuscript to a SpringerOpen ${ }^{\circ}$ journal and benefit from:}

- Convenient online submission

- Rigorous peer review

- Open access: articles freely available online

- High visibility within the field

- Retaining the copyright to your article

Submit your next manuscript at $\boldsymbol{\nabla}$ springeropen.com 